\title{
A New Algorithm for the Maximum Weighted Stable Set Problem in Claw-Free Graphs
}

\author{
Gianpaolo Oriolo ${ }^{1}$, Ugo Pietropaoli ${ }^{1}$, and Gautier Stauffer ${ }^{2}$ \\ ${ }^{1}$ Università di Roma "Tor Vergata", Dipartimento di Ingegneria dell'Impresa, \\ via del Politecnico 1, 00133 Roma, Italy \\ \{oriolo, pietropaoli\}@disp.uniroma2.it \\ ${ }^{2}$ IBM Research, Zurich Research Lab, Säumerstrasse 4, 8034 Rüschlikon, Switzerland \\ gst@zurich.ibm.com
}

\begin{abstract}
In this paper, we introduce two powerful graph reductions for the maximum weighted stable set (MWss) in general graphs. We show that these reductions allow to reduce the MWss in claw-free graphs to the MWss in a class of quasi-line graphs, that we call bipolar-free. For this latter class, we provide a new algorithmic decomposition theorem running in polynomial time. We then exploit this decomposition result and our reduction tools again to transform the problem to either a single matching problem or a longest path computation in an acyclic auxiliary graph (in this latter part we use some results of Pulleyblank and Shepherd [10]). Putting all the pieces together, the main contribution of this paper is a new polynomial time algorithm for the Mwss in clawfree graphs. A rough analysis of the complexity of this algorithm gives a time bound of $O\left(n^{6}\right)$, where $n$ is the number of vertices in the graph, and which we hope can be improved by a finer analysis. Incidentally, we prove that the Mwss problem can be solved efficiently for any class of graphs that admits a "suitable" decomposition into pieces where the MWSs is easy.
\end{abstract}

\section{Introduction}

A graph is claw-free if no vertex has a stable set of size three in its neighborhood. While the stable set problem is $\mathcal{N} \mathcal{P}$-hard in general, it can be solved in polynomial time for claw-free graphs 811126. The stable set problem on claw-free graphs is a fundamental generalization of the matching problem. In particular, from an algorithmic point of view, it generalizes the matching problem in two different ways, and all the current algorithms exploit either one of those two generalizations.

A first way to see this problem as a generalization of the matching problem is in terms of augmenting paths. Berge [1] proved that a matching $M$ is maximal for a graph $G$ if and only if there is no alternating path that is augmenting for $M$. This property, often called the augmenting path property, can be extended to stable sets in claw-free graphs as Berge also observed [2]. Indeed he proved that a stable set $S$ is maximal for a claw-free graph $G$ if and only if there is no 
alternating path that is augmenting for $S$. The algorithms by Sbihi [1], Minty [8] or the variation of Minty's algorithm given by Schrijver [12] are based on the detection of augmenting paths.

Another way to see the stable set problem in claw-free graphs as a generalization of the matching problem is in terms of graph structure. Indeed, line graphs are those graphs that can be obtained from a (possibly non simple) undirected graph $G$ by bijectively mapping the edges of $G$ to the vertices of a new graph $L(G)$ and by connecting two vertices in $L(G)$ if the corresponding edges are incident in $G$. It is straightforward to observe that for each vertex $v$ in $L(G), N(v)$ can be covered by two cliques. The graphs with this latter property are called quasi-line graphs. Quasi-line graphs (and thus line graphs) are claw-free. Now, due to the one-to-one mapping between the edges of a graph $G$ and the vertices of its line graph $L(G)$ and by definition of adjacencies in $L(G)$, there is also a one-to-one correspondence between matchings in $G$ and stable sets in $L(G)$ and thus the stable set problem in claw-free graphs is a generalization of the stable set problem in line graphs, which is a matching problem. Lovász and Plummer [6] defined graph reductions that preserve the stability number to reduce the unweighted stable set problem in a claw-free graph to an unweighted stable set problem in a line graph.

Unfortunately Lovász-Plummer's approach does not deal with the weighted version of the stable set problem on claw-free graphs. Therefore, the only algorithm for the weighted case is the one given in 1980 by Minty [8] (and the slightly different version discussed in Schrijver [12]). This algorithm is not very "natural", as it converts the original problem to the problem of detecting augmenting paths in some auxiliary graphs, called "Edmonds graphs", on which some matching problems are solved. Moreover, its overall complexity is $O\left(n^{6}\right)$, where $n$ denotes the number of vertices in the original graph 1 The question of finding a more direct and fast algorithm arises therefore naturally, as for instance it was recently pointed out in [7] "... one needs a better reduction from weighted claw-free to weighted line graphs, which seems to be a challenging research problem."

We also point out that an elegant algorithm has been given by Pulleyblank and Shepherd 10 for the maximum weighted stable set Mwss problem on a subclass of claw-free graphs, called distance claw-free. The algorithm is based on finding a longest path in an acyclic digraph and has complexity $O\left(n^{3}\right)$.

In this paper, we propose a new algorithm for the maximum weighted stable set (Mwss) problem in claw-free graphs. The algorithm is based on graph reductions and on a new decomposition theorem for a class of quasi-line graphs, that we call bipolar-free. First, we perform graph reductions that somehow extend the approach of Lovász and Plummer to the weighted case as to end up with an MWss problem on a bipolar-free quasi-line graph. We here use our decomposition theorem stating that a bipolar-free quasi-line graph that is not distance claw-free can be decomposed into at most $n$ distance claw-free graphs, that,

${ }^{1}$ In [7, it is claimed that the complexity of Minty's algorithm is $O\left(n^{7}\right)$; we have however not been able to convince ourselves that this is correct. 
because of their properties, we call distance simplicial. When the graph is distance claw-free, we use the algorithm by Pulleyblank and Shepherd [10] and otherwise, using our decomposition, it is possible to evaluate an Mwss for the original graph by solving a single matching problem. A rough analysis of the complexity of the proposed algorithm gives a time bound of $O\left(n^{6}\right)$, which we hope can be improved by a finer analysis, that we defer to the journal version of the paper. The steps of the algorithm are summarized below with pointers to the corresponding results in the paper.

\section{Sketch of the Algorithm}

1. Check that $G$ contains a stable set of size 4 by enumeration $\left(O\left(n^{4}\right)\right)$

- If not, find an Mwss by enumeration in $O\left(n^{3}\right)$ and stop.

2. Check that $G$ is quasi-line (by detecting 5 -wheels)

- If not, reduce the Mwss problem on $G$ to the Mwss problem on a graph $G^{\prime}$ which is either quasi-line or $\alpha\left(G^{\prime}\right) \leq 3\left(O\left(n^{4}\right)\right.$, see Lemma 13). If $\alpha\left(G^{\prime}\right) \leq 3$, solve the problem on $G^{\prime}$ by enumeration.

3. $G^{\prime}$ is a quasi-line graph (possibly, $G^{\prime}=G$ ). Check that $G^{\prime}$ is bipolar-free (by detecting bipolar pairs)

- If not, add appropriate edges to turn $G^{\prime}$ into a bipolar-free quasi-line graph $G^{\prime \prime}$ with the same vertex set and $\alpha_{w}\left(G^{\prime \prime}\right)=\alpha_{w}\left(G^{\prime}\right)\left(O\left(n^{6}\right)\right.$, see Lemma 17). Every stable set of $G^{\prime \prime}$ is also a stable set of $G^{\prime}$.

4. $G^{\prime \prime}$ is a bipolar-free quasi-line graph (possibly, $G^{\prime \prime}=G^{\prime}$ ). Check whether $G^{\prime \prime}$ has strongly regular articulation cliques $\left(O\left(n^{3}\right)\right.$, see Lemma 201)

- If not, $G^{\prime \prime}$ is distance claw-free: find an Mwss using the algorithm from [10] $\left(O\left(n^{3}\right)\right)$

- else decompose $G^{\prime \prime}$ into distance simplicial strips, evaluate the crucial stable sets for each of them, and solve a matching problem to re-combine them to an Mwss of $G^{\prime \prime}\left(O\left(n^{4}\right)\right.$, see Lemmas 31 and 5) and therefore of $G^{\prime}$.

The paper is organized as follows. In Section 2, we introduce two graph reductions for the Mwss in general graphs. In Section 3 we show how to use our first reduction to transform the Mwss problem on a claw-free graph to the same problem on a quasi-line graph. In Section 4 we show how to make use of our second reduction to reduce the MWss problem on quasi-line graphs to the MWss problem on bipolar-free quasi-line graphs. Finally, Section 5 is devoted to prove a decomposition theorem for this last class of graphs describing their structure, and to present the resulting algorithm for the MWss on the class of claw-free graphs.

\section{Definitions and Notations}

We close the introduction with some definitions and notations. Every graph $G(V, E)$ will be undirected and simple, i.e. without loops and parallel edges. The graph complement of $G$ is denoted by $\bar{G}$. A matching in $G$ is a set of edges that are pairwise non-incident. A stable set in $G$ is a set of vertices which are pairwise non-adjacent. The stability number of $G$ is the size of a stable set 
of maximum cardinality and it is denoted by $\alpha(G)$. Given a weight function $w: V \mapsto \mathbb{R}$, the weighted stability number is the weight of an Mwss in $G$ and is denoted by $\alpha_{w}(G)$. Given a set $U \subseteq V$, we denote by $G[U]$ the graph induced by the vertices in $U$. When no confusion can arise, we write $\alpha(U)$ and $\alpha_{w}(U)$ instead of $\alpha(G[U])$ and $\alpha_{w}(G[U])$, respectively. Moreover, we denote by $w(U)$ the sum of the weights of all vertices of $U$. A clique in $G$ is a set of pairwise adjacent vertices. We say that a clique $K$ is maximal if there does not exist a clique $K^{\prime} \supset K$.

Given a vertex $u \in V$, we denote by $N_{G}(u)$ the neighbors of $u$ in $G$, i.e. $N_{G}(u)$ $:=\{v \in V:(u, v) \in E\}$. When no confusion can arise, we denote $N_{G}(u)$ by $N(u)$. A vertex $u \in V$ is said to be universal to $v$ if $u$ is adjacent to $v$ and to every vertex in $N(v) \backslash\{u\}$; we denote by $U(v)$ the set of vertices that are universal to $v$. A vertex $u \in V$ is said to be simplicial if $N(u)$ is a clique. Given a set $U \subseteq V$, we denote by $E(U)$ the edges with both endpoints in $U$, by $\delta(U)$ the edges with one endpoint in $U$ and the other in $V(G) \backslash U$ and by $N(U)$ the set $\{v \in V \backslash U:(u, v) \in E$, for some $u \in U\}$. We also denote for all $j \geq 2, N_{j}(U):=N\left(N_{j-1}(U)\right) \backslash N_{j-2}(U)$ with the convention $N_{0}(U):=U$ and $N_{1}(U):=N(U)$. A vertex $u \in V$ is said to be strongly simplicial if $N(u)$ and $N_{2}(u)$ are cliques. Given two sets $U, U^{\prime} \subseteq V$, we denote by $E\left(U: U^{\prime}\right)$ the edges with one end in $U$ and the other in $U^{\prime}$. Two disjoint sets of vertices $A, B$ are said to be complete (resp. anticomplete) if $E(A: B)=\{(a, b): a \in A, b \in B\}$ (resp. $E(A: B)=\emptyset)$.

A path $P$ of length $k$ in $G(V, E)$ is an ordered sequence of edges $\left(e_{1}, \ldots, e_{k}\right)$ where $e_{i}=\left(v_{i}, v_{i+1}\right), v_{i} \in V$ for all $i=1, \ldots, k$ and $\left(v_{1}, v_{k+1}\right) \notin E$ (repetition of edges is not allowed). We denote $P=\left(e_{1}, \ldots, e_{k}\right)$. Since we consider simple graphs, we can also define $P$ by the ordered sequence of vertices $\left(v_{1}, \ldots, v_{k+1}\right)$ that are visited.

A claw $\left(c ; v_{1}, v_{2}, v_{3}\right)$ is a graph with vertex set $\left\{c, v_{1}, v_{2}, v_{3}\right\}$ and edge set $\left\{\left(c, v_{1}\right),\left(c, v_{2}\right),\left(c, v_{3}\right)\right\}$. A 5 -wheel $\left(c ; v_{1}, \ldots, v_{5}\right)$ is a graph with vertex set $\{c$, $\left.v_{1}, \ldots, v_{5}\right\}$ and edge set $\bigcup_{i=1}^{5}\left\{\left(c, v_{i}\right),\left(v_{i}, v_{i+1}\right)\right\}$ (with the convention $\left.v_{6}=v_{1}\right)$. A net $\left(u_{1}, u_{2}, u_{3} ; v_{1}, v_{2}, v_{3}\right)$ is a graph with vertex set $\left\{u_{1}, u_{2}, u_{3}, v_{1}, v_{2}, v_{3}\right\}$ and edge set $\left\{\left(v_{1}, v_{2}\right),\left(v_{2}, v_{3}\right),\left(v_{3}, v_{1}\right),\left(u_{1}, v_{1}\right),\left(u_{2}, v_{2}\right),\left(u_{3}, v_{3}\right)\right\}$. A gem $\left(v_{1}, v_{2}, v_{3}\right.$, $\left.v_{4}, v_{5}\right)$ is the graph with vertex set $\left\{v_{1}, \ldots, v_{5}\right\}$ and edge set $\left\{\left(v_{1}, v_{2}\right),\left(v_{1}, v_{3}\right)\right.$, $\left.\left(v_{2}, v_{3}\right),\left(v_{2}, v_{4}\right),\left(v_{3}, v_{4}\right),\left(v_{3}, v_{5}\right),\left(v_{4}, v_{5}\right)\right\}$.

Finally, for a set $S$, a family of subsets $\left\{S_{1}, . ., S_{k}\right\}$ is called a laminar family if, for $1 \leq i<j \leq k, S_{i} \cap S_{j} \neq \emptyset$ implies $S_{i} \subseteq S_{j}$ or $S_{j} \subseteq S_{i}$.

\section{Reductions for the Maximum Weighted Stable Set Problem}

\subsection{A Simple Reduction for Strips-Composed Graphs}

Chudnovsky and Seymour [3] introduced a composition operation in order to define their decomposition result for claw-free graphs. This composition procedure is general and applies to non-claw-free graphs as well. We borrow a couple 
of definitions from their work (even if our definition of gluing is slightly different). A strip $(G, a, b)$ is a graph (not necessarily connected) with two designated simplicial vertices $a$ and $b$. Observe that, by definition, if $a$ and $b$ are adjacent, then $N(a) \cup N(b)$ is a clique.

Given two vertex-disjoint strips $\left(G_{1}, a_{1}, b_{1}\right)$ and $\left(G_{2}, a_{2}, b_{2}\right)$, we define the gluing of those two strips as the union of $G_{1} \backslash\left\{a_{1}, b_{1}\right\}$ and $G_{2} \backslash\left\{a_{2}, b_{2}\right\}$ together with all edges between $N_{G_{1}}\left(a_{1}\right)$ and $N_{G_{2}}\left(a_{2}\right)$ and all edges between $N_{G_{1}}\left(b_{1}\right)$ and $N_{G_{2}}\left(b_{2}\right)$. Moreover, we add all edges between $N_{G_{1}}\left(a_{1}\right)$ and $N_{G_{1}}\left(b_{1}\right)$ when $a_{2}$ and $b_{2}$ are adjacent (and vice versa). Observe that this operation is closely related to the definition of 2 -join (cf. (4)). Note also that gluing $\left(G_{1}, a_{1}, b_{1}\right)$ and $\left(G_{2}, b_{2}, a_{2}\right)$ would not result in the same graph.

We can generalize the operation of gluing to several strips by introducing a composition operation.

Definition 1. Let $G^{0}$ be a disjoint union of cliques with $2 k$ vertices and $\left(G_{1}, a_{1}\right.$, $\left.b_{1}\right), \ldots,\left(G_{k}, a_{k}, b_{k}\right) k$ vertex disjoint strips with $\left(a_{i}, b_{i}\right) \notin E\left(G_{i}\right)$ for $i=1, \ldots, k$. Let $\phi$ be a one-to-one mapping from $\left\{a_{1}, \ldots, a_{k}, b_{1}, \ldots, b_{k}\right\}$ to $V\left(G^{0}\right)$. For all $i=1, \ldots, k$, define $G^{i}$ as the gluing of $\left(G_{i}, a_{i}, b_{i}\right)$ with $\left(G^{i-1}, \phi\left(a_{i}\right), \phi\left(b_{i}\right)\right)$. The graph $G^{k}$ is the composition of the strips $\left(G_{i}, a_{i}, b_{i}\right), i=1, \ldots, k$ w.r.t. $\left(G^{0}, \phi\right)$.

It is a simple exercise to prove that the composition does not depend on the order of the strips. In the following, we will refer to a graph that, as $G^{k}$, can be obtained by this procedure as a strips-composed graph. We will also use the following alternative definition for strips-composed graphs.

Definition 2. Let $\left(G_{1}, a_{1}, b_{1}\right), \ldots,\left(G_{k}, a_{k}, b_{k}\right)$ be $k$ vertex disjoint strips with $\left(a_{i}, b_{i}\right) \notin E\left(G_{i}\right)$ for $i=1, \ldots, k$ and let $\mathcal{P}:=\left\{P_{1}, \ldots, P_{m}\right\}$ be a partition of the vertices $\left\{a_{1}, \ldots, a_{k}, b_{1}, \ldots, b_{k}\right\}$. Let $G^{0}$ be the union of the graphs $G_{i} \backslash\left\{a_{i}, b_{i}\right\}$, $i=1, \ldots, k$. For all $j=1, \ldots, m$, define $G^{j}$ as the graph obtained from $G^{j-1}$ by adding all edges between $N(u)$ and $N(v)$ for all $u, v \in P_{j}$. The graph $G^{m}$ is the composition of the strips $\left(G_{i}, a_{i}, b_{i}\right), i=1, \ldots, k$ w.r.t. the partition $\mathcal{P}$.

It is again a simple exercise to prove that the composition does not depend on the order of the classes in the partition. It is also easy to prove that the two definitions are equivalent, i.e. they define the same class of graphs, and that we can pass easily from one representation to the other. Indeed, the disjoint cliques of $G^{0}$ (together with $\phi$ ) define a natural partition of the vertices $\left\{a_{1}, \ldots, a_{k}, b_{1}, \ldots, b_{k}\right\}$ and from a partition it is easy to define a suitable set of disjoint cliques and a mapping. We skip the details. In both cases, we say that $\left(G_{i}, a_{i}, b_{i}\right), i=1, \ldots, k$ define a strip decomposition, either of $G^{k}$ w.r.t. $\left(G^{0}, \phi\right)$ or for $G^{m}$ w.r.t. $\mathcal{P}$.

The different strips involved in the composition can be complex objects. Nevertheless, when we are dealing with stable set problems, there is a simple reduction that allows to get rid of non desirable strips (e.g. non-line strips).

Lemma 3. Let $G$ be the gluing of two strips $(H, a, b)$ and $\left(H^{\prime}, c, d\right)$ where $(a, b) \notin$ $E(H)$. Let $w: V \mapsto \mathbb{R}$ be a weight function on the vertices of $G$. Let $G^{\prime}$ be the gluing of $\left(T, v_{1}, v_{5}\right)$ and $\left(H^{\prime}, c, d\right)$, where $\left(T, v_{1}, v_{5}\right)$ is a gem. Let $A:=N_{H}(a)$, 
$B:=N_{H}(b), C=N_{H^{\prime}}(c)$ and $D=N_{H^{\prime}}(d)$ and define a weight function $w^{\prime}$ for $G^{\prime}$ as follows:

$-w^{\prime}(v)=w(v)$ if $v \in V\left(H^{\prime} \backslash\{c, d\}\right)$

$-w^{\prime}\left(v_{2}\right):=w_{\bar{B}}-w_{\overline{A \cup B}}$

$-w^{\prime}\left(v_{3}\right)=w_{H}-w_{\overline{A \cup B}}$

$-w^{\prime}\left(v_{4}\right):=w_{\bar{A}}-w_{\overline{A \cup B}}$.

where $w_{H}$ is the weight of an Mwss in $H \backslash\{a, b\}$ if $(c, d) \notin H^{\prime}$ or the weight of an MWSS in $H \backslash\{a, b\}$ picking at most one vertex in $A \cup B$ if $(c, d) \in H^{\prime}$, while $w_{\bar{B}}$ (resp. $w_{\bar{A}}, w_{\overline{A \cup B}}$ ) is the weight of an MWss in $H \backslash\{a, b\}$ not intersecting $B$ (resp. $A, A \cup B$ ). Then $\alpha_{w^{\prime}}\left(G^{\prime}\right)=\alpha_{w}(G)-w_{\overline{A \cup B}}$.

Proof. Let $S$ be an mwss in $G$. We have to deal with four cases. Either $S$ does not pick any vertex in $C \cup D$ or it picks a vertex in $D$ but not in $C$, or it picks a vertex in $C$ but not in $D$, or it picks a vertex in $C$ and a vertex in $D$ (possibly the same if $C \cap D \neq \emptyset)$.

Let us analyse the first situation. We claim that $w(S \cap V(H))=w_{H}$. If $c$ and $d$ are not adjacent, $S \cap V(H)$ must be an Mwss in $V(H) \backslash\{a, b\}$ : otherwise, if $w_{S \cap V(H)}<w_{H}=w\left(S_{H}\right)$, for some suitable stable set $S_{H} \subseteq V(H) \backslash\{a, b\}$, $S_{H} \cup\left(S \cap V\left(H^{\prime}\right)\right)$ would be a better stable set for $G$ (it is a stable set, since there are no adjacencies between $V(H)$ and $V\left(H^{\prime}\right) \backslash(C \cup D)$ in $G$ and there is no new adjacency in $G[V(H)])$. If $c$ and $d$ are adjacent, then $S \cap V(H)$ takes at most one vertex in $A \cup B$ (by definition $A$ is complete to $B$ in $G$ in that case) and must thus be an mWss in $V(H) \backslash\{a, b\}$ picking at most one vertex in $A \cup B$ for the same reasons.

Now in $G^{\prime}, S^{\prime}=\left\{v_{3}\right\} \cup\left(S \cap V\left(H^{\prime}\right)\right)$ is a stable set (since $S$ does not intersect $C \cup D)$. But $w^{\prime}\left(S^{\prime}\right)=w^{\prime}\left(S \cap V\left(H^{\prime}\right)\right)+w^{\prime}\left(v_{3}\right)=w\left(S \cap V\left(H^{\prime}\right)\right)+w_{H}-w_{\overline{A \cup B}}=$ $w\left(S \cap V\left(H^{\prime}\right)\right)+w(S \cap V(H))-w_{\overline{A \cup B}}=w(S)-w_{\overline{A \cup B}}$. Hence $\alpha_{w^{\prime}}\left(G^{\prime}\right) \geq \alpha_{w}(G)-$ $w \overline{A \cup B}$. The three other cases can be analyzed similarly.

Conversely, let $S^{\prime}$ be an Mwss in $G^{\prime}$. Again we have to deal with the four cases above. Let us analyze the case where $S^{\prime}$ does not intersect $C \cup D$ : in this case, w.l.o.g., we may assume that $v_{3} \in S^{\prime}$ (since $w^{\prime}\left(v_{4}\right) \leq w^{\prime}\left(v_{3}\right) \geq w^{\prime}\left(v_{2}\right)$ ). Thus let $S^{\prime \prime}$ be an Mwss in $H \backslash\{a, b\}$ (picking at most one vertex in $A \cup B$ if $c$ and $d$ are adjacent). $S=S^{\prime \prime} \cup\left(S^{\prime} \cap V\left(H^{\prime}\right)\right)$ is a stable set in $G$. But $w(S)=w\left(S^{\prime \prime}\right)+w\left(S^{\prime} \cap V\left(H^{\prime}\right)\right)=w_{H}+w^{\prime}\left(S^{\prime} \cap V\left(H^{\prime}\right)\right)=w^{\prime}\left(v_{3}\right)+w_{\overline{A \cup B}}+$ $w^{\prime}\left(S^{\prime} \cap V\left(H^{\prime}\right)\right)=w^{\prime}\left(S^{\prime}\right)+w_{\overline{A \cup B}}$. Thus $\alpha_{w^{\prime}}\left(G^{\prime}\right) \leq \alpha_{w}(G)-w_{\overline{A \cup B}}$. Again the three other cases can be analyzed similarly.

Let $(H, a, b)$ be a strip and let $S_{H}$ be an Mwss in $H \backslash\{a, b\}$ (picking at most one vertex in $A \cup B$ if $c$ and $d$ are adjacent), $S_{\bar{B}}$, (resp. $S_{\bar{A}}, S_{\overline{A \cup B}}$ ) an Mwss in $H \backslash\{a, b\}$ not intersecting $B$ (resp. $A, A \cup B$ ). We say that $S_{H}, S_{\bar{B}}, S_{\bar{A}}, S_{\overline{A \cup B}}$ are the crucial Mwss for $(H, a, b)$.

Remark 4. From the proof of the above lemma not only one can get $\alpha_{w}(G)$ from $\alpha_{w^{\prime}}\left(G^{\prime}\right)$, but also one can build an Mwss for $G$ from an Mwss for $G^{\prime}$ in constant time, if the crucial Mwss for $(H, a, b)$ are given. 
We have a result similar to Lemma 3 for strips-composed graphs.

Lemma 5. Let $G$ be the composition of the strips $\left(G_{i}, a_{i}, b_{i}\right), i=1, \ldots, k$ w.r.t. a partition $\mathcal{P}$. Suppose that the crucial MWss can be computed in time $O\left(p_{i}(n)\right)$ for the different strips $i=1, \ldots, k$ ( $n$ being the number of vertices of $G$ ). Then the Mwss problem on $G$ can be solved in time $O\left(\sum_{i=1, \ldots . k} p_{i}(n)+\right.$ match $\left.(n)\right)$, where match $(n)$ is the time required to solve a matching problem on a graph with $n$ vertices. If $p_{i}(n)$ is polynomial for each $i$, then the Mwss problem can be solved in polynomial time.

Proof. First of all, as we pointed out above, we can equivalently express $G$ as the composition of the strips $\left(G_{i}, a_{i}, b_{i}\right), i=1, \ldots, k$, w.r.t. some pair $\left(G^{0}, \phi\right)$. We now use the procedure defined in Lemma 3 and associate to each strip $\left(G_{i}, a_{i}, b_{i}\right)$ a gem $\left(T^{i}, v_{1}^{i}, v_{5}^{i}\right)$ with suitable weights. Let $H$ be the composition of the strips $\left(T^{i}, v_{1}^{i}, v_{5}^{i}\right), i=1, \ldots, k$ w.r.t. $\left(G^{0}, \phi\right)$. For each strip $i$, let $w_{\overline{A_{i} \cup B_{i}}}$ be the weight of an MWSs in $G_{i} \backslash\left\{a_{i}, b_{i}\right\}$ not intersecting $A_{i} \cup B_{i}$, where $A_{i}=N_{G_{i}}\left(a_{i}\right)$ and $B_{i}=N_{G_{i}}\left(b_{i}\right)$.

Claim 6. $\alpha_{w}(H)=\alpha_{w}(G)-\sum_{i=1, \ldots, k} w_{\overline{A_{i} \cup B_{i}}}$.

Proof. $G$ is the composition of $\left(G_{1}, a_{1}, b_{1}\right), \ldots,\left(G_{k}, a_{k}, b_{k}\right)$ w.r.t. $\left(G^{0}, \phi\right)$. Let $G^{\prime}$ be the composition of $\left(G_{1}, a_{1}, b_{1}\right), \ldots,\left(G_{k-1}, a_{k-1}, b_{k-1}\right),\left(T^{k}, v_{1}^{k}, v_{5}^{k}\right)$ w.r.t. $\left(G^{0}, \phi\right)$. It follows from Lemma 3 that $\alpha_{w}(G)=\alpha_{w}\left(G^{\prime}\right)+w_{\overline{A_{k} \cup B_{k}}}$. Recall that the composition does not depend on the order of the strips, i.e. $G^{\prime}$ is also the composition of $\left(G_{1}, a_{1}, b_{1}\right), \ldots,\left(G_{k-2}, a_{k-2}, b_{k-2}\right),\left(T^{k}, v_{1}^{k}, v_{5}^{k}\right),\left(G_{k-1}, a_{k-1}\right.$, $\left.b_{k-1}\right)$ w.r.t. $\left(G^{0}, \phi\right)$. We now define $G^{\prime \prime}$ to be the composition of $\left(G_{1}, a_{1}, b_{1}\right), \ldots$, $\left(G_{k-2}, a_{k-2}, b_{k-2}\right),\left(T^{k}, v_{1}^{k}, v_{5}^{k}\right),\left(T^{k-1}, v_{1}^{k-1}, v_{5}^{k-1}\right)$ w.r.t. $\left(G^{0}, \phi\right)$. We have that $\alpha_{w}\left(G^{\prime}\right)=\alpha_{w}\left(G^{\prime \prime}\right)+w_{\overline{A_{k-1} \cup B_{k-1}}}$. The claim follows by iterating this reasoning. (End of the claim.)

Claim 7. $H$ is the line graph of a graph $F$ and $F$ can be built in time $O(k)$.

Proof. First, note that $H$ has $3 k$ vertices. $G^{0}$ is a graph with $2 k$ vertices that is the disjoint union of $p$ cliques. By definition, each clique of $G^{0}$ induces a clique of $H$. We then consider the family $\mathcal{K}$ of cliques of $H$ that is made of the $p$ previous cliques together with the cliques $\left\{v_{2}^{1}, v_{4}^{1}\right\}, \ldots,\left\{v_{2}^{k}, v_{4}^{k}\right\}$. It is easy to see that $\mathcal{K}$ covers all the edges of $H$ and that every vertex of $H$ belongs to exactly 2 cliques of $\mathcal{K}$. It is shown in [6] that, in this case, $H$ is the line graph of a graph $F$, that can be built as follows. For each clique $K$ in $\mathcal{K}$, we associate a vertex $v_{K}$ in $F$. For all vertices $v_{K} \neq v_{K^{\prime}}$, we add $\left|K \cap K^{\prime}\right|$ edges $\left(v_{K}, v_{K^{\prime}}\right)$. Observe that we can build $F$ directly from $G$, i.e. we do not need to build $H$, in time $O(k)$, since $|V(F)|=k+p \leq 3 k$ and $|E(F)|=|V(H)|=3 k$. (End of the claim.)

We are thus left with solving a weighted matching problem in $F$. The weights of the edges of $F$ (vertices of $H$ ) can be given in time $O\left(\sum_{i=1, \ldots, k} p_{i}(n)\right)$. Moreover, it follows by induction from Remark 4 that an Mwss of $G$ can be built in time $O(k)$ from an Mwss of $H$, i.e. a maximum weighted matching of $F$. Observe that $k=O(n)$. The statement follows. 
We want to stress again that Lemma 3 and Lemma 5 apply not only to claw-free graphs, but to all graphs that can be obtained as the composition of strips.

\subsection{Semi-homogeneous Pairs of Cliques}

The notion of semi-homogeneous pair of cliques extend the classical concept of homogeneous pair of cliques (a pair of cliques $(A, B)$ is homogeneous if for all $x \in V \backslash\{A \cup B\}, x$ is either complete to $A \cup B$ or anti-complete to $A \cup B$ or complete to $A$ (resp. $B$ ) and anti-complete to $B$ (resp. $A$ )).

Definition 8. A pair of cliques $(A, B)$ in a graph $G(V, E)$ is semi-homogeneous if for all $x \in V \backslash\{A \cup B\}, x$ is either complete to $A$ or complete to $B$ or anticomplete to $A \cup B$.

Lemma 9. Let $G=(V, E)$ be a graph with a weighted function $w$ on its vertices. Let $(A, B)$ be a semi-homogeneous pair of cliques. Let $\{a, b\}$ be an MWSS of size two in $A \cup B$. Adding any edge different from $(a, b)$ between a vertex of $A$ and vertices of $B$ does not change $\alpha_{w}$; moreover, every stable set of the new graph is a stable set of $G$ with the same weight.

Proof. Suppose that $S$ is an Mwss of $G$ picking a vertex $a^{\prime}$ in $A$ and a vertex $b^{\prime}$ in $B$. For all $s \in S \backslash\left\{a^{\prime}, b^{\prime}\right\}, s$ is neither complete to $A$ nor to $B$. Thus since $(A, B)$ is semi-homogeneous, $s$ is anti-complete to $A \cup B$. Therefore we could replace $a^{\prime}$ by $a$ and $b^{\prime}$ by $b$ and getting a stable set of weight as big.

We will use the previous lemma to get rid of some "annoying" pairs of vertices in quasi-line graphs, that we call bipolar. In fact we will show that if a quasi-line graph has a bipolar pair of vertices, then it contains a semi-homogeneous pair of cliques $(A, B)$ such that there are at least two missing edges between $A$ and $B$.

\section{From Claw-free Graphs to Quasi-Line Graphs}

Recall that a graph $G$ is quasi-line if, for all $v$ in $V(G), N(v)$ can be covered by two cliques, that is, $\overline{G[N(v)]}$ is bipartite. Therefore, a claw-free graph $G$ is not quasi-line if and only if there exists a vertex $v$ with an odd-hole in $\overline{G[N(v)]}$.

While claw-free graphs with small stability number can be significantly different from quasi-line graphs, Fouquet [5] proved that claw-free graphs with stability number greater than 3 do not differ that much from quasi-line graphs.

Lemma 10. [5] A connected claw-free graph $G$ with $\alpha(G) \geq 4$ that does not contain a 5-wheel is quasi-line.

We have now a couple of lemmas whose proofs we defer to the journal version of the paper. The first one deals with the gluing operation that we defined in the previous section for general graphs. When we restrict to claw-free graphs, this operation preserves the structure of the graph. 
Lemma 11. The gluing of two claw-free strips is a claw-free graph.

The second lemma shows that a connected claw-free $G$, that is not quasi-line and has stability number at least four, is indeed the gluing of two claw-free strips.

Lemma 12. Let $G(V, E)$ be a connected claw-free graph with $\alpha(G) \geq 4$. Let $W:=\left(a ; u_{1}, u_{2}, u_{3}, u_{4}, u_{5}\right)$ be a 5-wheel. Then $G$ is the gluing of two clawfree strips $\left(H_{1}, a_{1}, b_{1}\right)$ and $\left(H_{2}, a_{2}, b_{2}\right)$ where $W$ is a subgraph of $H_{1}, \alpha\left(G\left[H_{1} \backslash\right.\right.$ $\left.\left.\left\{a_{1}, b_{1}\right\}\right]\right) \leq 3$ and $\left(a_{1}, b_{1}\right) \notin E\left(H_{1}\right)$. The two graphs $H_{1}$ and $H_{2}$ can be built in time $O\left(n^{3}\right)$.

Lemma 13. Let $G(V, E, w)$ be a claw-free graph, with $n$ vertices and $w: V \mapsto \mathbb{R}$. There exists a claw-free graph $\tilde{G}(\tilde{V}, \tilde{E}, \tilde{w})$, with $\tilde{w}: \tilde{V} \mapsto \mathbb{R}$ such that:

$-\alpha_{w}(G)=\alpha_{\tilde{w}}(\tilde{G})$ and $|\tilde{V}| \leq n$

- either $\tilde{G}$ is quasi-line or $\alpha(\tilde{G}) \leq 3$.

Moreover, the graph $\tilde{G}$ can be built in time $O\left(n^{4}\right)$, while an Mwss for $G$ can be built in time $O(n)$ from one in $\tilde{G}$.

Proof. The statement is trivial if either $G$ is quasi-line or $\alpha(G) \leq 3$, so suppose $G$ is not quasi-line and with $\alpha(G) \geq 4$. There must exist a vertex $v$ with an odd-hole in $\overline{G[N(v)]}$. Indeed detecting an odd-hole in a graph $H$ that is trianglefree is standard $(\overline{G[N(v)]}$ is triangle-free since $G$ is claw-free) and can be done in $O(m)$. We need to visit an auxiliary graph $H^{\prime}$, where each vertex $u \in V(H)$ is duplicated into $u^{\prime}, u^{\prime \prime}$ and, for each edge $(u, v) \in E(H)$, we add two edges $\left(u^{\prime}, v^{\prime \prime}\right)$ and $\left(u^{\prime \prime}, v^{\prime}\right)$ in $H^{\prime}$. It is not difficult to see that a path of minimum length connecting $u^{\prime}$ to $u^{\prime \prime}$ corresponds, after shrinking duplicated vertices, to a shortest odd hole in $H$. Since $G$ is not quasi-line, in time $O(n m)$ we must detect an odd-hole in some $\overline{G[N(v)]}$ : we take the smallest one. This has to be a 5 -hole by Lemma 10.

So we have detected a 5 -wheel in $G$. Using Lemma 12, we build in time $O\left(n^{3}\right)$ the claw-free strips $\left(H_{1}, a_{1}, b_{1}\right)$ and $\left(H_{2}, a_{2}, b_{2}\right)$, where $H_{1}$ has an induced subgraph that is a 5 -wheel and $\left(a_{1}, b_{1}\right) \notin E\left(H_{1}\right)$. We replace $H_{1}$ by a gem and define the graph $G^{\prime}\left(V^{\prime}, E^{\prime}, w^{\prime}\right)$ that is the gluing of $\left(H_{2}, a_{2}, b_{2}\right)$ with the gem, as

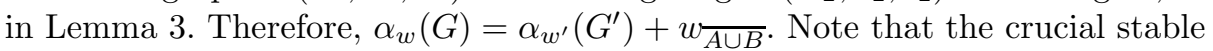
sets for $H_{1}$, and therefore the weights $w^{\prime}$ on the vertices of the gem, can be computed in $O\left(n^{3}\right)$ by enumeration since $\alpha\left(G\left[H_{1} \backslash\left\{a_{1}, b_{1}\right\}\right]\right) \leq 3 . G^{\prime}$ is claw-free because of Lemma 11.

Observe that, all together, $G^{\prime}$ can be built in time $O\left(n^{3}\right)$ from $G$. Now observe that $G^{\prime}$ contains less vertices (at least two less) than $G$. If either $\alpha\left(G^{\prime}\right) \leq 3$ or $G^{\prime}$ is quasi-line, we stop and let $\tilde{G}=G^{\prime}$, else we repeat this procedure (at most $O(n)$ times since we remove at least two vertices each time). Therefore, $\tilde{G}$ can be built in time $O\left(n^{4}\right)$ from $G$ and has less than $n$ vertices. Finally, we may build an MWss of $G$ from an MWss of $\tilde{G}$ in time $O(n)$ by applying inductively Remark 4. The statement follows. 
We are now left to solve the stable set problem in quasi-line graphs. Even if quasi-line graphs are more friendly from a structural point of view than clawfree graphs, there is a configuration that always annoyed us and can be easily removed when dealing with the stable set problem: bipolar pairs of vertices.

\section{From Quasi-Line Graphs to Bipolar-Free Quasi-Line Graphs}

Lovász and Plummer [6] called regular a vertex of a claw-free graph such that $\{v\} \cup N(v)$ can be covered by two maximal cliques. We say that $v$ is strongly regular when this covering is unique. Recall that $U(v)$ is the set of vertices that are universal to some vertex $v$.

Lemma 14. A vertex $v$ of a quasi-line graph $G(V, E)$ is strongly regular if and only if $H=\overline{G[N(v) \backslash U(v)]}$ is connected. In this case, $\left(H_{1} \cup U(v) \cup\{v\}, H_{2} \cup\right.$ $U(v) \cup\{v\})$ is the unique pair of maximal cliques covering $\{v\} \cup N(v)$, where $\left(H_{1}, H_{2}\right)$ are the classes of the (unique) bi-coloring of $V(H)$.

Definition 15. Given a quasi-line graph $G$ and two vertices $v_{1}, v_{2}$ that are not universal to each other (i.e., $v_{1}$ is not universal to $v_{2}$ and $v_{2}$ is not universal to $\left.v_{1}\right)$, we say that they form a bipolar pair if there exists a maximal clique $K$ such that $v_{1}, v_{2} \in K$ and $\left(N\left(v_{1}\right) \cup N\left(v_{2}\right)\right) \backslash K$ is a clique.

A quasi-line graph without bipolar pairs of vertices is said to be bipolar-free. The notion of bipolar pair of vertices for quasi-line graphs is closely related to the more general concept of semi-homogeneous pair of cliques that we defined in Section 2.2, as it is confirmed by the following lemma.

Lemma 16. Let $G$ be a quasi-line graph and let $\left(v_{1}, v_{2}\right)$ be a bipolar pair. Let $A=\left\{v_{1}, v_{2}\right\}$ and $B=\left(N\left(v_{1}\right) \backslash\left(N\left(v_{2}\right) \cup\left\{v_{2}\right\}\right)\right) \cup\left(N\left(v_{2}\right) \backslash\left(N\left(v_{1}\right) \cup\left\{v_{1}\right\}\right)\right)$. Then $(A, B)$ is a semi-homogeneous pair of cliques in $G$.

Proof. We have to prove that a vertex $v \notin A \cup B$ that is neither complete to $A$ nor to $B$ is anticomplete to $A \cup B$. By hypothesis, $\left(v_{1}, v_{2}\right)$ is a bipolar pair, i.e. there exists a maximal clique $K$ such that $v_{1}, v_{2} \in K$ and $\left(N\left(v_{1}\right) \cup N\left(v_{2}\right)\right) \backslash K$ is a clique. Assume w.l.o.g. that $v$ is not adjacent to $v_{2}$. In particular, $v \notin K$. Moreover, $v$ is not adjacent to $v_{1}$, since otherwise, $v \in\left(N\left(v_{1}\right) \backslash N\left(v_{2}\right)\right) \subseteq B$. Assume that $v$ is not anti-complete to B, then there exists $w$ w.l.o.g. in $N\left(v_{2}\right) \backslash N\left(v_{1}\right)$ that is adjacent to $v$ ( $w$ exists since $v_{1}$ is not universal to $v_{2}$ ). But then $v$ is complete to $N\left(v_{1}\right) \backslash N\left(v_{2}\right)$. Indeed suppose that there is $z \in N\left(v_{1}\right) \backslash N\left(v_{2}\right)$ not adjacent to $v$, then $\left(w ; z, v_{2}, v\right)$ is a claw. But we can apply the same reasoning with $w \in N\left(v_{1}\right) \backslash N\left(v_{2}\right)$ to show that $v$ is complete to $N\left(v_{2}\right) \backslash N\left(v_{1}\right)$. Thus $v$ is complete to $B$ a contradiction.

Lemma 17. Let $G(V, E, w)$ be a quasi-line graph with $n$ vertices and $w: V \mapsto$ $\mathbb{R}$. There exists a bipolar-free quasi-line graph $G^{\prime}\left(V, E^{\prime}, w\right)$, with $E^{\prime} \supseteq E$, such that $\alpha_{w}(G)=\alpha_{w}\left(G^{\prime}\right)$. Moreover, the graph $G^{\prime}$ can be built in time $O\left(n^{6}\right)$. 
Proof. Let $w: V \mapsto \mathbb{R}$ be the weight function on the vertices of $G$. Our procedure goes as follows. We detect a bipolar pair of vertices for $G$, if any. If $G$ has no bipolar pairs of vertices, we stop. Else, let $v_{1}, v_{2}$ be a bipolar pair for $G$. We know from Lemma 16 that $A=\left\{v_{1}, v_{2}\right\}$ and $B=\left(N\left(v_{1}\right) \backslash\left(N\left(v_{2}\right) \cup\left\{v_{2}\right\}\right)\right) \cup$ $\left(N\left(v_{2}\right) \backslash\left(N\left(v_{1}\right) \cup\left\{v_{1}\right\}\right)\right)$ form a semi-homogeneous pair of clique. Without loss of generality assume that $\left\{v_{2}, w_{1}\right\}$ is an Mwss of size two in $A \cup B$. We define a graph $G^{\prime}$ by adding to $G$ every edge different from $\left(v_{2}, w_{1}\right)$ between a vertex of $A$ and vertices of $B$. We know from Lemma 9 that $\alpha_{w}(G)=\alpha_{w}\left(G^{\prime}\right)$ and every stable set of $G^{\prime}$ is a stable set of $G$ with the same weight. The graph $G^{\prime}$ is still quasi-line (see the following). If $G^{\prime}$ has no more bipolar pairs of vertices, we stop; else we iterate. Since we cannot add more than $O\left(n^{2}\right)$ edges to $G$, we iterate at most $O\left(n^{2}\right)$ times.

Our proof follows by induction if we prove that:

1 Detecting a bipolar pair of vertices of a quasi-line graph $G$, if any, can be done in time $O\left(n^{4}\right)$.

$2 G^{\prime}$ is still quasi-line.

1. Let $u$ and $v$ be a pair of vertices that are not universal to each other. It is straightforward to see that they form a bipolar pair if and only if $\overline{G[N(u) \cup N(v)]}$ is bipartite and admits a bi-coloring where $u$ and $v$ get the same color. Therefore, we can detect a bipolar pair of vertices in time $O\left(n^{4}\right)$.

2. We consider the case where $G^{\prime}=G \cup\left(v_{1}, w_{2}\right)$, i.e. $A$ is complete to $B$ but for the edges $\left\{v_{2}, w_{1}\right\}$ and $\left(v_{1}, w_{2}\right)$ (the general case follows easily by induction).

We want to show that $G^{\prime}$ is still quasi-line, i.e. the neighborhood of each vertex can be covered by two cliques. Observe that, by definition, $E(G) \subset E\left(G^{\prime}\right)$ and, by hypothesis, $G$ is quasi-line. Therefore, for any vertex $v \notin\left\{v_{1}, w_{2}\right\}, N_{G^{\prime}}(v)$ can be still covered by two cliques, since $N_{G^{\prime}}(v)=N_{G}(v)$. As far as $v_{1}$ and $w_{2}$ are concerned, it will be enough to show that:

(i) there exist two cliques of $G$ covering $N_{G}\left(v_{1}\right) \cup\left\{w_{2}\right\}$;

(ii) there exist two cliques of $G$ covering $N_{G}\left(w_{2}\right) \cup\left\{v_{1}\right\}$.

First, we need some definitions and a claim. Let $K_{1}\left(K_{2}\right.$, resp.) be a maximal clique such that $K$ and $K_{1}$ ( $K$ and $K_{2}$, resp.) cover $N\left(v_{1}\right)\left(N\left(v_{2}\right)\right.$, resp.).

Denote by $S_{1}, S_{2}, \ldots, S_{8}$ a partition of the vertex set $V(G)$ of $G$, defined as follows: $S_{1}=\left\{v: v \in\left(K \cap K_{1}\right) \backslash K_{2}\right\}, S_{2}=\left\{v: v \in\left(K \cap K_{2}\right) \backslash K_{1}\right\}, S_{3}$ $=\left\{v: v \in K_{2} \backslash\left(K_{1} \cup K\right)\right\}, S_{4}=\left\{v: v \in K_{1} \backslash\left(K_{2} \cup K\right)\right\}, S_{5}=\{v: v \in$ $\left.K \cap K_{1} \cap K_{2}\right\}, S_{6}=\left\{v: v \in\left(K_{1} \cap K_{2}\right) \backslash K\right\}, S_{7}=\left\{v: v \in K \backslash\left(K_{1} \cup K_{2}\right)\right\}, S_{8}$ $=\left\{v: v \in V \backslash\left(K \cup K_{1} \cup K_{2}\right)\right\}$.

Claim 18. If there exists $z \in S_{8}$ such that $\left(z, w_{2}\right) \in E$ or $\left(z, w_{1}\right) \in E$, then $z$ is complete to $S_{3}$ and to $S_{4}$.

Proof. W.l.o.g. suppose $\exists z \in S_{8}$ s.t. $\left(z, w_{2}\right) \in E$. First we prove that $z$ is complete to $S_{4}$. In fact, $S_{4}, z, v_{2} \subseteq N\left(w_{2}\right)$. Moreover $S_{4} \cup\{z\}$ is anticomplete to $v_{2}$. Thus, $S_{4} \cup\{z\}$ must be a clique, since $G$ is quasi-line. Now we prove that $z$ 
is complete to $S_{3}$. In fact, $S_{3}, z, v_{1} \subseteq N\left(w_{1}\right)$. Moreover $S_{3}$ is not complete to $v_{1}$ and $z$ is not adjacent to $v_{1}$. Thus, $S_{3} \cup\{z\}$ must be a clique, since $G$ is quasi-line. (End of the claim.)

(i). From the above definitions, $N_{G}\left(v_{1}\right)=S_{1} \cup S_{5} \cup S_{2} \cup S_{7} \cup S_{4} \cup S_{6}$. Since $S_{1} \cup S_{5} \cup S_{2} \cup S_{7}=K$ and $S_{4} \cup S_{6} \cup\left\{w_{2}\right\}$ are both cliques of $G$, by hypothesis or construction, the statement follows.

(ii). We have: $N_{G}\left(w_{2}\right)=S_{3} \cup S_{6} \cup S_{4} \cup S_{2} \cup S_{5} \cup \tilde{S}_{1} \cup \tilde{S}_{7} \cup \tilde{S}_{8}$, where $\tilde{S}_{i}$ denotes the set of vertices belonging to $S_{i}$ that are adjacent to $w_{2}$. Observe that $v_{1}$ is complete to $S_{6} \cup S_{2} \cup S_{5} \cup \tilde{S}_{1} \cup \tilde{S}_{7}$. Therefore, in order to prove our statement, it is enough to show that there exist two cliques $K_{L}, K_{R}$ of $G$ covering $N_{G}\left(w_{2}\right)$ and such that $S_{3} \cup S_{4} \cup \tilde{S}_{8}$ belongs to a same clique, say $K_{L}$. This is the same as showing that there is a valid bi-coloring of the bipartite graph $H=\overline{G\left[N_{G}\left(w_{2}\right)\right]}$ such that $S_{3}, S_{4}, \tilde{S}_{8}$ get the same color, which for our purposes will be either red or blue. In the following, we build such a coloring.

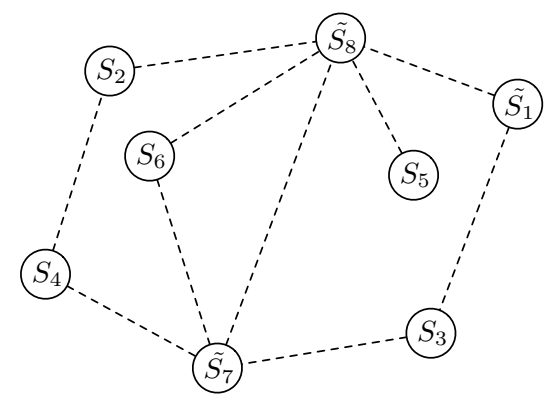

Fig. 1. The possible adjacencies in $H$. Two sets are connected by a dotted edge if and only if they are not complete to each other in $G . S_{4}$ and $S_{3}$ are complete by hypothesis; $\tilde{S}_{8}$ is complete to $S_{3} \cup S_{4}$ by Claim 18, other pairs that are complete belong to a same clique in $\left\{K, K_{1}, K_{2}\right\}$.

The graph $H$ is in general not connected and in Fig. 1 we represent the possible adjacencies in $H$ among the sets $\tilde{S}_{1}, S_{2}, S_{3}, S_{4}, S_{5}, S_{6}, \tilde{S}_{7}, \tilde{S}_{8}$, i.e. two sets are connected by a dotted edge if and only if they are not complete to each other in $G$. Consider the component $C_{1}$ in $H$ containing $v_{2}$ and say $v_{2}$ is blue. The set $S_{4}$ and $\tilde{S}_{8}$ are anticomplete in $G$ to $v_{2}$ and thus they are in $C_{1}$ and they have to be red. By maximality of the clique $K_{1}$, each vertex in $S_{2}$ has a non-neighbor in $S_{4}$ and thus $S_{2}$ is in $C_{1}$ and has to be blue. Consider a vertex $v \in S_{5}$. By definition, $v$ is complete to each $S_{i}$ but, possibly, $S_{8}$. Therefore, if $v$ is complete to $\tilde{S}_{8}$, then it is a singleton for $H$ and thus can be made arbitrarily blue; else $v$ belongs to $C_{1}$ and it is again blue.

So far we have seen that there exists a valid partial bi-coloring of $H$ such that w.l.o.g. $S_{4}$ and $\tilde{S}_{8}$ are red while $S_{2}$ and $S_{5}$ are blue. We now show that also the vertices of $S_{3}$ that are in $C_{1}$ are red. Observe that, since $H$ is bipartite and $C_{1}$ 
is connected, the coloring of the vertices of $C_{1}$ is forced: vertices that have even distance from the vertices in $S_{4} \cup \tilde{S}_{8}$ are red, vertices that have odd distance from the vertices in $S_{4} \cup \tilde{S}_{8}$ are blue; therefore it is enough to show that no vertex of $S_{3} \cap V\left(C_{1}\right)$ has odd distance from some vertex in $S_{4} \cup \tilde{S}_{8}$. Therefore, suppose to the contrary that $s_{3} \in S_{3}$ and $x \in S_{4} \cup \tilde{S}_{8}$ have odd distance and assume w.l.o.g. their distance to be minimum. Let $P=\left(s_{3}, u_{2}, u_{3}, \ldots, u_{2 k} \equiv x\right)$ be a path attaining such minimum distance.

We base our analysis on the possible edges in $H$ from the graph of Fig. 1. Recall that the sets $\tilde{S}_{1}, S_{2}, S_{3}, S_{4}, S_{5}, S_{6}, \tilde{S}_{7}$ are cliques for $G$ (and therefore stable sets for $H$ ). It is clear that $P$ does not contain any vertex of $\tilde{S}_{1}$ (since $P$ is of minimum distance between $S_{3}$ and $S_{4} \cup \tilde{S}_{8}$, in this case it would be a path of length 2 from $S_{3}$ to $\tilde{S}_{8}$ ). Analogously, $P$ cannot take any vertex from $S_{4}$, else $P$ would be an even path from $s_{3}$ to $S_{4}$, and it cannot take any edge from $\tilde{S}_{7}$ to $S_{8}$, else $P$ would be an even path from $S_{3}$ to $\tilde{S}_{8}$. It is thus clear that $P$ alternates between vertices of $\tilde{S}_{7}$ and $S_{6}$ before ending with a vertex of $S_{8}$ : namely, $u_{3}, u_{5}, \ldots, u_{2 k-1} \in S_{6}$ and $u_{2}, u_{4}, \ldots, u_{2 k-2} \in \tilde{S}_{7}$. Let $V(P)$ be the vertices in $P$; it follows that the vertices in $V(P) \cap S_{6}$ are blue while the vertices in $V(P) \cap \tilde{S}_{7}$ are red. Therefore, in $H$ there cannot be adjacencies between vertices in $S_{4}$ and vertices in $V(P) \cap \tilde{S}_{7}$, that is, vertices in $S_{4}$ are complete to vertices in $V(P) \cap \tilde{S}_{7}$ in $G$. Now observe that $P$ is of minimum distance, hence it is an induced odd path of $H$. The subgraph of $H$ induced by $V(P) \cup\left\{v_{1}\right\}$ is an induced odd-hole $\left(v_{1}\right.$ is complete in $G$ to $S_{6}, \tilde{S}_{7}$ and anticomplete to $\tilde{S}_{8}$ and $\left.S_{3}\right)$. Therefore $G\left[V(P) \cup\left\{v_{1}\right\}\right]$ is an induced anti-hole. But $\left(V(P) \cup\left\{v_{1}\right\}\right) \subseteq N_{G}\left(w_{1}\right)$. Indeed $w_{1}$ is complete to $S_{6}, v_{1}, S_{3}$ by definition, to $\tilde{S}_{8}$ by the previous claim and, since it belongs to $S_{4}$, it is complete to $V(F) \cap \tilde{S}_{7}$ as we have just observed. It follows that $N_{G}\left(w_{1}\right)$ contains an odd antihole, but this is in contradiction with $G$ being quasi-line. Therefore, the vertices of $S_{3}$ that are in $C_{1}$ are red.

Finally, let $Q$ be the set of vertices of $H$ that are not in $C_{1}$ and are not from $S_{5}$. It follows from above that $Q \subseteq \tilde{S}_{1} \cup \tilde{S}_{7} \cup S_{3} \cup S_{6}$. Clearly, any coloring for the vertices of $Q$ that is valid for $H[Q]$ is also valid for $H$. In particular, we may give color red to the vertices of $Q \cap\left(S_{3} \cup S_{6}\right)$ and color blue to the vertices in $Q \cap\left(\tilde{S}_{1} \cup \tilde{S}_{7}\right)$.

We have therefore built a valid bi-coloring for $H$ where $S_{3}, S_{4}, \tilde{S}_{8}$ get the same color (red) and statement (ii) is proved.

From Lemma 13 and Lemma 17, we know that in order to solve the Mwss problem in claw-free graphs, we only need to be able to solve the Mwss problem in bipolar-free quasi-line graphs. We will now analyze the structure of those graphs in order to devise a polynomial time algorithm.

\section{A Decomposition Theorem for Bipolar-Free Quasi-Line Graphs}

In this section we give our main structural result, concerning the structure of bipolar-free quasi-line graphs. In particular, we will show that a rich class of 
bipolar-free quasi-line graphs is the composition of suitable strips that can be found by identifying articulation cliques.

Definition 19. A maximal clique $K$ of a graph $G$ is an articulation clique if, for each $v \in K, N(v) \backslash K$ is a clique.

An articulation clique $K$ of a quasi-line graph $G(V, E)$ is called strongly regular if each vertex $v \in K$ is strongly regular. Observe that $K$ is a strongly regular articulation clique if and only if, for each $v \in K$, there is a unique pair of maximal cliques covering $N(v) \cup\{v\}$ and $K$ is one of these cliques. Detecting if a quasi-line graph has a strongly regular articulation clique is therefore easy.

Lemma 20. One can check in time $O\left(n^{3}\right)$ if a quasi-line graph $G(V, E)$ has a strongly regular articulation clique. Actually, one can list all strongly regular articulation cliques in time $O\left(n^{3}\right)$.

Proof. In order to detect a strongly regular articulation clique, we first build the set $R$ of strongly regular vertices and, for each vertex $v \in R$, the unique pair of maximal cliques $\left(K_{1}(v), K_{2}(v)\right)$ covering $N(v) \cup\{v\}$. That can be done in time $O\left(n^{3}\right)$, thanks to Lemma 14. Let $K(R)=\left\{K_{1}(v), K_{2}(v), v \in R\right\}$. A clique $K$ of $G$ is a strongly regular articulation one if and only if $K \subseteq R$ and, for each $v \in K, K \in\left\{K_{1}(v), K_{2}(v)\right\}$. Since $|K(R)| \leq 2 n$, it follows that we can list all strongly regular articulation cliques in time $O\left(n^{3}\right)$.

Definition 21. A maximal clique $K$ is a net clique if there exists a stable set of size three $\left\{s_{1}, s_{2}, s_{3}\right\}$ in $N(K)$ and each vertex in $K$ is adjacent to at most one vertex in $\left\{s_{1}, s_{2}, s_{3}\right\}$.

Lemma 22. In a quasi-line graph, a net clique $K$ is a strongly regular articulation clique.

Proof. It is enough to show that, for every vertex $v \in K$, there exists a maximal clique $K(v)$ such that $(K, K(v))$ is the unique covering of $N(v)$ into two maximal cliques.

By definition, there exists a stable set $\left\{s_{1}, s_{2}, s_{3}\right\} \subseteq N(K)$ and each $v \in K$ is adjacent to at most one vertex in $\left\{s_{1}, s_{2}, s_{3}\right\}$. So let $K_{1}=K \cap N\left(s_{1}\right), K_{2}=$ $K \cap N\left(s_{2}\right), K_{3}=K \cap N\left(s_{3}\right), K_{4}=K \backslash\left(K_{1} \cup K_{2} \cup K_{3}\right)\left(K_{1}, K_{2}, K_{3} \neq \emptyset\right.$ since $\left.\left\{s_{1}, s_{2}, s_{3}\right\} \subseteq N(K)\right)$.

First, suppose $v \in K_{1}$. Let $\left(Q_{1}, Q_{2}\right)$ be a pair of maximal cliques such that $N(v) \cup\{v\}=Q_{1} \cup Q_{2}$ (such a pair exists, since the graph is quasi-line). Assume w.l.o.g. that $s_{1} \in Q_{1}$, it follows that $K \backslash K_{1} \subseteq Q_{2}$. We now show that every vertex $z \in N(v) \backslash K$ is not complete to $K \backslash K_{1}$. Suppose the contrary, i.e. there exists $z \in N(v) \backslash K$ that is complete to $K \backslash K_{1}$. Since $K$ is maximal, there exists $w \in K_{1}, w \neq v$, such that $(w, z) \notin E$. Since $z$ is adjacent to $v$, it cannot be adjacent to both $s_{2}$ and $s_{3}$ (otherwise there would be the claw $\left(z ; s_{2}, s_{3}, v\right)$ ). Assume w.l.o.g. $z$ is not linked to $s_{3}$. Let $z_{3}$ be a vertex in $K_{3}$. Then $\left(z_{3} ; s_{3}, w, z\right)$ 
is a claw, a contradiction. Therefore, every vertex in $z \in N(v) \backslash K$ is not complete to $K \backslash K_{1}$ and so it must belong to $Q_{1}$. It follows that $Q_{1}=(N(v) \backslash K) \cup\{v\} \cup U(v)$ and $Q_{2}=K$, that is, $\left(Q_{1}, K\right)$ is the unique covering of $N(v)$ into two maximal cliques. The same holds for any vertex $v$ in $K_{2}$ or $K_{3}$.

Now suppose that $v \in K_{4}$. If $v$ is a simplicial vertex, then the statement is trivial. Now suppose that there exists $w \notin K$ such that $(w, v) \in E$. Observe that $w$ is adjacent to at most one vertex of $\left\{s_{1}, s_{2}, s_{3}\right\}$ : if the contrary, assume w.l.o.g. $s_{1}, s_{2} \in N(w)$, there would be the claw $\left(w ; v, s_{1}, s_{2}\right)$. Hence there exists a stable set of size three in $\left\{w, s_{1}, s_{2}, s_{3}\right\}$ containing $w$ and we are back to the previous case.

Definition 23. Let $K$ be an articulation clique. For every $v \in K$, we define:

$$
Q(v)=\left\{\begin{array}{ll}
\{v\} & \text { if } v \text { is simplicial } \\
Q(v)=U(v) \cup\{v\} & \text { else }
\end{array} .\right.
$$

Moreover, we let $\mathcal{Q}(K)$ be the family of inclusion-wise maximal cliques in $\{Q(v)$, $v \in K\}$ and say that $v$ generates $Q$, for some $Q \in \mathcal{Q}(K)$, if $Q=Q(v)$.

Lemma 24. Let $G(V, E)$ be a quasi-line graph and $K \subseteq V$ an articulation clique. The following statements hold:

(i) For each $v \in K, v \in Q(v) \subseteq K$.

(ii) For each $u, v \in K$, if $u \in Q(v)$ then $Q(u) \subseteq Q(v)$.

(iii) If $G$ is bipolar-free, then $\mathcal{Q}(K)$ defines a partition of $K$.

(iv) If $G$ is bipolar-free and $Q \in \mathcal{Q}(K)$, then $N(Q) \backslash K$ is a clique.

Proof. Let $v$ be a vertex of $K$. (i) It is trivial if $v$ is simplicial. Else, let $u$ be a vertex in $N(v) \backslash K$. Since $K$ is maximal, there exists some vertex $z \in K$ such that $(u, z) \notin E$; therefore $u$ is not universal to $v$ and does not belong to $Q(v)$. (ii) Suppose that $u, v \in V, u \neq v$. Observe that $v$ is not simplicial. The statement is trivial if $u$ is simplicial. So assume that it is not. Let $z \in Q(u)$, we want to show that $z \in Q(v)$. It is trivial if $z \equiv u$, so assume that $z \neq u$. By definition, $N(u) \cup\{u\} \subseteq N(z) \cup\{z\}$. On the other hand, since $u \in Q(v)$, $N(v) \cup\{v\} \subseteq N(u) \cup\{u\}$. Therefore, $N(v) \cup\{v\} \subseteq N(z) \cup\{z\}$. (iii) It follows from (i) that, in order to show that $\mathcal{Q}(K)$ defines a partition of $K$, it is enough to show that the family $\{Q(v), v \in K\}$ is laminar. Suppose to the contrary that that there exist $u, v$ with $Q(v) \cap Q(u), Q(v) \backslash Q(u), Q(u) \backslash Q(v) \neq \emptyset$. Therefore $u$ and $v$ are not simplicial and it follows from (ii) that $v \notin Q(u)$ and $u \notin Q(v)$. That is, $u$ and $v$ are not universal to each other. Therefore, there exists $z$ such that $(z, v) \in E$ and $(z, u) \notin E$. Similarly, there exists $y$ such that $(y, u) \in E$ and $(y, v) \notin E$. Let $w \in Q(v) \cap Q(u)$. Observe that $N(u) \backslash K$ and $N(v) \backslash K$ belong to $N(w)$, since $w$ is universal to $u$ and $v$. On the other hand, $K$ is an articulation clique, therefore $N(w) \backslash K$ is a clique. It follows that $(N(u) \cup N(v)) \backslash K$ is a clique. Then $(u, v)$ is a bipolar pair, a contradiction. (iv) Suppose the contrary. There exist $x, y \in N(Q) \backslash K$ such that $(x, y) \notin E$. Since $K$ is an articulation 
clique, it follows that no vertex of $Q$ is joined to both $x$ and $y$. Therefore there exist $u, v \in Q$ such that $(u, y) \in E,(u, x) \notin E,(v, x) \in E,(v, y) \notin E$. Neither $u$ nor $v$ can generate $Q$, as $(u, y) \in E$ and $(u, x) \notin E$, as well as $(v, x) \in E$ and $(v, y) \notin E$. Therefore there exists $w \in K$ that generates $Q$. Such a vertex $w$ is not simplicial (otherwise it could not generate $Q$ ), thus there exists a vertex $n \notin K$ such that $(n, w) \in E$. The neighbors of $w$ outside $K$ must be universal to $u$ and $v$, thus $(n, u) \in E$ and $(n, v) \in E$ (therefore $n \notin\{x, y\})$. For the same reason $x, y \notin N(w)$. Finally, $(n, x) \in E$ and $(n, y) \in E$, otherwise $u$ or $v$ would be vertices of $K$ with a stable set of size 2 in their neighborhood outside $K$. It follows that $(n ; x, y, w)$ is a claw, which is a contradiction.

Definition 25. Let $G$ be a bipolar-free quasi-line graph and $K$ an articulation clique with $\mathcal{Q}(K)=\left\{Q_{1}, \ldots, Q_{k}\right\}$. The ungluing of the articulation clique $K$ produces the graph $G_{\mid K}$, defined as follows:

1. Remove all the edges between vertices of $Q_{i}$ and vertices of $Q_{j}$, for $i, j \in$ $\{1, \ldots, k\}, i \neq j$.

2. Add a set $\mathcal{A}(K):=\left\{a_{1}, \ldots, a_{k}\right\}$ of artificial vertices. For each $i \in\{1, \ldots, k\}$, let $N_{G_{\mid K}}\left(a_{i}\right)=Q_{i}$.

We defer to the journal version of this paper the proof of the following lemma, showing that the ungluing operation preserves quasi-lineness, bipolar-freeness and has other useful properties.

Lemma 26. Let $K$ be an articulation clique in a connected bipolar-free quasiline graph $G$. The following statements hold:

(i) $G_{\mid K}$ is quasi-line.

(ii) $G_{\mid K}$ is bipolar-free.

(iii) In each component of $G_{\mid K}$ there is a vertex from $G$ and a vertex from $\mathcal{A}(K)$. Moreover each vertex from $\mathcal{A}(K)$ is strongly simplicial and all vertices of $\mathcal{A}(K)$ are pairwise non-adjacent in $G_{\mid K}$.

(iv) If $w \notin K$ is simplicial for $G$, then $w$ is simplicial for $G_{\mid K}$ too.

\subsection{Distance Simplicial Graphs}

Pulleyblank and Shepherd [10] showed that, given a fixed $k$, the Mwss problem can be solved via longest paths in an acyclic digraph in time $O\left(n^{k+1}\right)$ for connected graphs with a vertex $v$ having $\alpha\left(N_{j}(v)\right) \leq k$ for all $j$. This motivated them to define distance claw-free a connected graph such that, for every $v$ and every $j, \alpha\left(N_{j}(v)\right) \leq 2$. Trivially, distance claw-free graphs are a subclass of claw-free graphs and it follows from what above that one can solve the Mwss problem in distance claw-free graphs in time $O\left(n^{3}\right)$. They also proved that a connected claw-free graph that is not distance claw-free has an induced net. We have therefore the following lemma, whose proof is omitted.

Lemma 27. Let $G$ be a connected claw-free graph. If $G$ is not distance claw-free then it has an induced net clique. 
Definition 28. A connected graph $G(V, E)$ is distance simplicial if there exists a vertex $v \in V$ such that $N_{j}(v)$ is a clique for each $j$.

It follows from above that the Mwss problem can be solved in time $O\left(n^{2}\right)$ for distance simplicial graphs. It is possible to show that a distance simplicial graph is distance claw-free, but, since we do not need this statement for the following, we defer it and its proof to the journal version of this paper.

We also defer the proof of the following lemma, showing that a claw-free graph with a strongly simplicial vertex is either distance simplicial or has a net-clique $K$, that we can use to unglue $G$. Moreover, $G_{\mid K}$ has some useful properties.

Lemma 29. Let $G(V, E)$ be a connected claw-free graph with a strongly simplicial vertex $a$. If $G$ is not distance simplicial, then it has an induced net clique $K$, where $K=N_{j-1}(a) \cup H$, with $H \subseteq N_{j-2}(a)$, for some $j \geq 3$. Moreover, the following statements hold:

(i) $K$ can be found in time $O\left(n^{2}\right)$.

(ii) No vertex of $K$ is strongly simplicial.

(iii) If $G$ is quasi-line and bipolar-free, then $G_{\mid K}$ is not connected.

(iv) If $G$ is quasi-line and bipolar-free and $a^{\prime}$ is a strongly simplicial vertex not adjacent to a (possibly, $a^{\prime} \equiv a$ ), then $a^{\prime}$ is strongly simplicial in $G_{\mid K}$ too.

\subsection{The Decomposition Algorithm}

We are now ready to define our decomposition procedure (cf. Algorithm 1). The algorithm receives a connected graph $G$ that is quasi-line and bipolar-free, but not distance claw-free. It returns: a graph $G^{L}$ (still quasi-line and bipolar-free) such that $V\left(G^{L}\right)=V(G) \cup A^{L}$ (the vertices in $A^{L}$ are artificial) and such that each component is distance simplicial; a partition $\mathcal{P}$ of the vertices in $A^{L}$. As we show later, the components of $G^{L}$ and the partition $\mathcal{P}$ can be used to define a strip decomposition of $G$.

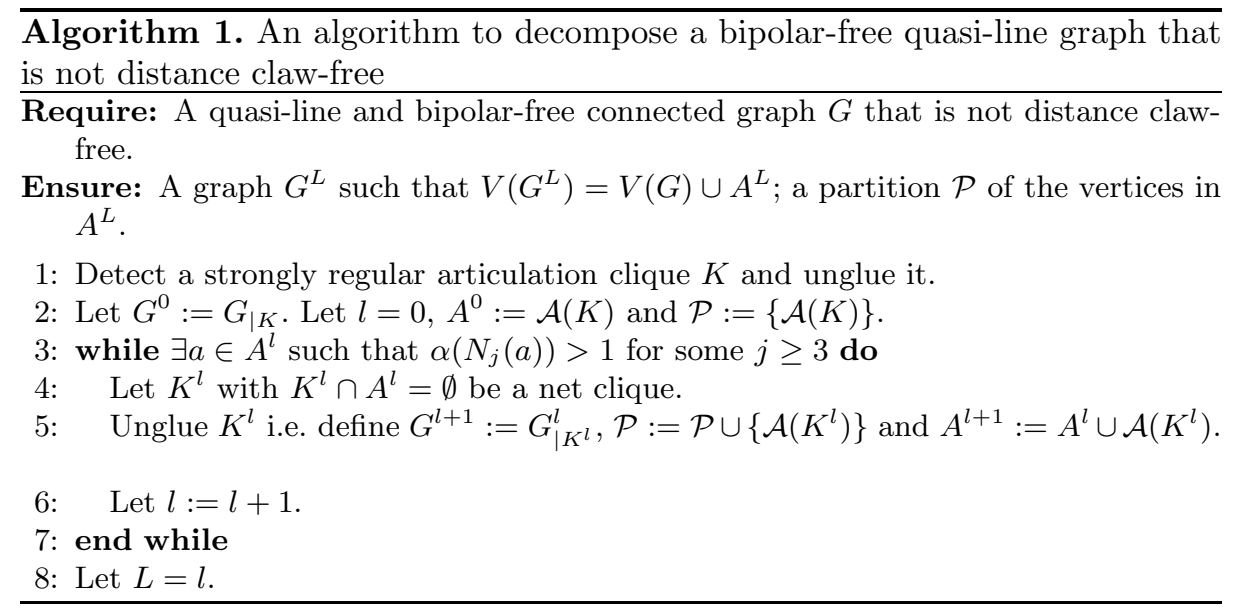


In the algorithm, we start with a connected graph $G$ that is quasi-line and bipolar-free but not distance claw-free. Hence $G$ has a net clique by Lemma 27. and therefore a strongly regular articulation clique $K$ by Lemma 22. We unglue $K$ and set $A^{0}$ equal to the set of artificial vertices $\mathcal{A}(K)$. By statements $(i)$ and (ii) of Lemma 26, $G^{0}:=G_{\mid K}$ is quasi-line and bipolar-free, moreover, by statement (iii), the vertices in $A^{0}$ are pairwise non-adjacent, every vertex of $A^{0}$ is strongly simplicial and in every component of $G^{0}$ there is a vertex from $A^{0}$ and a vertex from $G$.

We then check whether in some component of $G^{0}$ there exists a vertex $a \in A^{0}$ such that $\alpha\left(N_{j}(a)\right)>1$ for some $j$. If not, by Lemma 29, each component of $G^{0}$ is distance simplicial and the algorithm terminates; else, there exists a net clique $K^{0}$, that we unglue, setting $G^{1}:=G_{\mid K^{0}}^{0}$ and $A^{1}:=A^{0} \cup \mathcal{A}\left(K^{0}\right)$. Observe that, by Lemma 29. no vertex of $K^{0}$ is strongly simplicial, therefore $K^{0} \cap A^{0}=\emptyset$, $G^{1}$ has at least one component more than $G^{0}$ and each vertex of $A^{0}$ is strongly simplicial in $G^{1}$ (we can use the fourth statement of Lemma 29, since the vertices in $A^{0}$ are pairwise non-adjacent).

By Lemma 26, $G^{1}$ is quasi-line, bipolar-free, the vertices in $\mathcal{A}\left(K^{0}\right)$ are pairwise non-adjacent and every vertex of $\mathcal{A}\left(K^{0}\right)$ is strongly simplicial in $G_{1}$. Also in every component of $G_{1}$ there is a vertex of $A^{1}$ and a vertex from $G$ (about this last statement, observe that each new component has a vertex from $K^{0}$ and $K^{0} \cap A^{0}=\emptyset$ ). Moreover the vertices in $A^{1}$ are still pairwise non-adjacent: we have already seen that vertices in $A^{0}$ are pairwise non-adjacent and vertices in $\mathcal{A}\left(K^{0}\right)$ are pairwise non-adjacent. Now observe that $A^{0}$ is anti-complete to $\mathcal{A}\left(K^{0}\right)$ : this is because the only adjacencies defined during the reduction of $K^{0}$ are adjacencies between vertices of $\mathcal{A}\left(K^{0}\right)$ and vertices of $K^{0}$ and no vertex of $A^{0}$ belongs to $K^{0}$.

We set $l=1$ and iterate. It follows, by induction, that at each step $l$ of the algorithm: the graph $G^{l}$ is quasi-line and bipolar-free; the set $A^{l}$ is made of pairwise non-adjacent strongly simplicial vertices; in each component of $G^{l}$ there is at least one vertex from $A^{l}$ and at least one vertex from $G$. Therefore, at each step, unless each component of $G^{l}$ is distance simplicial, there is a net clique to unglue. The algorithm will terminate after $L$ iterations when no component of $G^{L}$ has a vertex $a \in A^{L}$ such that $\alpha\left(N_{j}(a)\right)>1$ for some $j$, i.e. when each component of $G^{L}$ is distance simplicial. In particular, since we cannot have more than $n=|V(G)|$ components with at least a vertex from $G$, it follows that $L \leq n$. Moreover, since each iteration can be done in time $O\left(n^{3}\right)$, the running time of the algorithm is $O\left(n^{4}\right)$.

We finally show how the components of $G^{L}$ and $\mathcal{P}$ can be used to define a strip decomposition of $G$. Observe that, by construction, $V\left(G^{L}\right)=V(G) \cup A(L)$ and $\mathcal{P}$ defines a partition of the vertices in $A^{L}$.

Lemma 30. Each component of $G^{L}$ has at most two vertices from $A^{L}$.

Proof. Suppose to the contrary that there exists a connected component $C$ in $G^{L}$ with more than two vertices from $A^{L}$. Let $a_{1}, a_{2}, a_{3} \in C \cap A^{L}$. In the following, we refer to the graph $G^{L}$. Recall that each vertex in $A^{L}$ is strongly simplicial 
and that the vertices in $A^{L}$ are pairwise non-adjacent. Assume $a_{2} \in N_{j}\left(a_{1}\right)$ and $a_{3} \in N_{k}\left(a_{1}\right)$ for some $k \geq j \geq 1$. Actually, $j>1$ since otherwise $a_{1}$ and $a_{2}$ would be adjacent. Since each component of $G^{L}$ is distance simplicial, it follows that $\alpha\left(N_{l}\left(a_{1}\right)\right) \leq 1$ for all $l \geq 1$. Therefore, $k>j$ and thus $N_{j+1}\left(a_{1}\right) \neq \emptyset$. Each vertex $v \in N_{j+1}\left(a_{1}\right)$ is not adjacent to $a_{2}$ (else $a_{2}$, that is adjacent to some vertex in $N_{j-1}\left(a_{1}\right)$, would not be simplicial). But then since there exists $w \in N_{j-2}\left(a_{1}\right) \cap N_{2}\left(a_{2}\right)$, it follows that $\{v, w\}$ is a stable set of size two in $N_{2}\left(a_{2}\right)$, a contradiction.

Suppose now that $G^{L}$ has $p$ components $H_{1}, \ldots, H_{p}$. Each component $H_{i}$, for $i=1$..p , has either two vertices $a_{i}, b_{i}$ from $A^{L}$ or one vertex $a_{i}$ from $A^{L}$. W.l.o.g. assume that each component has two artificial vertices (else we might add a singleton to the component). Consider therefore the strips $\left(H_{1}, a_{1}, b_{1}\right), \ldots,\left(H_{p}\right.$, $\left.a_{p}, b_{p}\right)$. It is easy to see that $G$ is the composition of the strips $\left(H_{i}, a_{i}, b_{i}\right), i=$ $1, \ldots, p$ w.r.t. the partition $\mathcal{P}$ : in fact, $G^{L}, G^{L-1}, \ldots, G^{0}$ is exactly the sequence defined in the alternative definition of strips-composed graph (see Def. 21).

We can summarize our previous results as follows:

Lemma 31. Let $G$ be a connected and bipolar-free quasi-line graph $G$ that is not distance claw-free. By Algorithm 11 we can produce a strip decomposition of $G$ in time $O\left(n^{4}\right)$.

Theorem 32. Let $G$ be a connected bipolar-free quasi-line graph.

- Either $G$ is a distance claw-free graph without strongly simplicial vertices and without strongly regular articulation cliques;

- or $G$ is the composition of at most $n$ distance simplicial strips.

We now have all the ingredients to state our main result.

Theorem 33. The Mwss problem can be solved in time $O\left(n^{6}\right)$ for a claw-free graph $G$.

Proof. We check if there exists a stable set of size 4 in $G$ by enumeration: this can be done in time $O\left(n^{4}\right)$. If not, we enumerate in time $O\left(n^{3}\right)$ all stable sets of $G$ of size 1, 2 and 3 and take the best one. Else, if the graph has stability number greater than 3, we use Lemma 13 to reduce to the Mwss problem in a quasi-line graph $G^{1}$ in time $O\left(n^{4}\right)$. We now use Lemma 17 to reduce to the Mwss problem in a bipolar-free quasi-line graph $G^{2}$ in time $O\left(n^{6}\right)$. We search for strongly regular articulation cliques in $G^{2}$ : this can be done in time $O\left(n^{3}\right)$ from Lemma 20, If there are no strongly regular articulation cliques, then we know from Lemma 22 that $G^{2}$ has no net cliques and therefore (Lemma 27) $G^{2}$ is distance claw-free; hence we use the algorithm of Pulleyblank and Shepherd [10] and find an Mwss in time $O\left(n^{3}\right)$. Else, we get a strip decomposition of $G^{2}$ into distance simplicial strips from Lemma 31 in time $O\left(n^{4}\right)$. The crucial stable sets can be found in each strip in time $O\left(n^{2}\right)$ (first recall that in a distance simplicial graph an Mwss can be found in time $O\left(n^{2}\right)$, then observe that each crucial stable set is an Mwss, if we give weight 0 to some suitable set of vertices). Therefore, from Lemma [5, we can find an Mwss in $G^{2}$ in time $O\left(n^{3}\right)$. All together, we can solve the Mwss problem for $G$ in time $O\left(n^{6}\right)$. 


\section{Acknowledgment}

Defining graph reductions that can deal with the MWSS problem requires a deep understanding of the structure of claw-free graphs. Recently, Chudnovsky and Seymour (see the survey paper [3]) tackled this problem and they provided decomposition theorems for claw-free and quasi-line graphs. Though we started considering graph reductions only after we heard about this tremendous piece of work, we could finally avoid to use their whole machinery thanks to our simpler decomposition result we could prove for bipolar-free quasi-line graphs. We believe nevertheless that we would not have thought about our simpler decomposition without the decomposition theorems of Chudnovsky and Seymour.

\section{References}

1. Berge, C.: Two theorems in graph theory. Proc. Nat. Acad. Sci. U.S.A. 43, 842-844 (1957)

2. Berge, C.: Graphs and hypergraphs. Dunod, Paris (1973)

3. Chudnovsky, M., Seymour, P.: The structure of claw-free graphs. In: Surveys in Combinatorics 2005. London Math. Soc. Lecture Note Series, vol. 327 (2005)

4. Cornuéjols, G., Cunningham, W.H.: Compositions for perfect graphs. Discrete Mathematics 55, 245-254 (1985)

5. Fouquet, J.: A strengthening of Ben Rebea's lemma. Journal of Combinatorial Theory 59, 35-40 (1993)

6. Lovász, L., Plummer, M.D.: Matching theory. North Holland, Amsterdam (1986)

7. Lozin, V.V., Milanič, M.: A polynomial algorithm to find an independent set of maximum weight in a fork-free graph. In: Proceedings of SODA 2006, Miami, Florida, January 22-26 (2006)

8. Minty, G.J.: On maximal independent sets of vertices in claw-free graphs. Journal of Combinatorial Theory 28, 284-304 (1980)

9. Nakamura, D., Tamura, A.: A revision of Minty's algorithm for finding a maximum weighted stable set of a claw-free graph. Journal of the Operations Research Society of Japan 44(2), 194-204 (2001)

10. Pulleyblank, W., Shepherd, F.: Formulations for the stable set polytope. In: Rinaldi, G., Wolsey, L.A. (eds.) Proceedings of IPCO 1993, Erice, Italy, April 19 May 1 (1993)

11. Sbihi, N.: Algorithme de recherche d'un stable de cardinalité maximum dans un graphe sans étoile. Discrete Mathematics 29, 53-76 (1980)

12. Schrijver, A.: Combinatorial optimization. Polyhedra and efficiency. In: Algorithms and Combinatorics 24, 3 volumes. Springer, Berlin (2003) 